\title{
Non-POSIX File System for LHCb Online Event Handling
}

\author{
Jean-Christophe Garnier, Niko Neufeld, and S. S. Cherukuwada
}

\begin{abstract}
LHCb aims to use its $\mathrm{O}(20000)$ CPU cores in the high level trigger (HLT) and its 120 TB Online storage system for data reprocessing during $\mathrm{LHC}$ shutdown periods. These periods can last a few days for technical maintenance or only a few hours during beam interfill gaps. These jobs run on files which are staged in from tape storage to the local storage buffer. The result are again one or more files. Efficient file writing and reading is essential for the performance of the system. Rather than using a traditional shared file-system such as NFS or CIFS we have implemented a custom, light-weight, non-Posix network file-system for the handling of these files. Streaming this file-system for the data-access allows to obtain high performance, while at the same time keep the resource consumption low and add nice features not found in NFS such as high-availability, transparent fail-over of the read and write service. The writing part of this streaming service is in successful use for the Online, real-time writing of the data during normal data acquisition operation. The network file system relies on a commercial file system which manages the data on persistent disk storage. The implementation together with performance figures are presented.
\end{abstract}

Index Terms-Data acquisition, fail-over, high-availability, network file-system.

\section{INTRODUCTION}

$\mathbf{T}$ HE LHCb [1] experiment at CERN is dedicated to the study of CP-violation, a subtle asymmetry in the fundamental laws of nature, which is thought to be responsible for the striking abundance of matter over anti-matter in the observable universe. When the LHCb detector enters in operation, dedicated Data Acquisition boards read fragments of events from sub-detector electronics and send these fragments to the High Level Trigger (HLT) [2] farm. In the farm, these fragments are assembled to build the complete event. Events are approximatively $35 \mathrm{kB}$ each and are pushed into the HLT farm at a $1 \mathrm{MHz}$ rate. Events are then analyzed by trigger algorithms which determine whether they should be discarded or recorded. Selected events are consolidated into files in the $\mathrm{LHCb}$ onsite storage. The output rate of the trigger algorithms is expected to be $2 \mathrm{kHz}$, with peaks up to $5 \mathrm{kHz}$. The data rate at which events have to be written to the storage system varies from $70 \mathrm{MB} / \mathrm{s}$ to $170 \mathrm{MB} / \mathrm{s}$.

Manuscript received June 11, 2010; revised November 04, 2010; accepted February 07, 2011. Date of publication March 17, 2011; date of current version August 17, 2011. This work was supported by a Marie Curie Initial Training Network Fellowship of the European Community's Seventh Framework Programme under Contract (PITN-GA-2008-211801-ACEOLE).

The authors are with CERN, Geneva CH-1211, Switzerland (e-mail: jeanchristophe.garnier@cern.ch; niko.neufeld@cern.ch).

Color versions of one or more of the figures in this paper are available online at http://ieeexplore.ieee.org.

Digital Object Identifier 10.1109/TNS.2011.2116804
To optimize resource usage, the HLT farm could be used for event reprocsesing [3] during LHC [4] shutdown periods. It would perform the same task as the one performed by the Worldwide LHC Computing Grid (WLCG) [5], but with a different implementation as it is subject to different constraints. On the Grid, one core processes one complete file. A file of $2 \mathrm{~GB}$ contains about 60000 events and an event is reprocessed in one or two seconds. This means that a core needs approximately one day to reprocess a file. Moreover we would need at least 20000 files to give a task to each core. It is rather slow and storage consuming, which is unacceptable for our system. In addition, the LHC shutdown periods can vary from a few hours ${ }^{1}$ to a few days. ${ }^{2}$ So we have to adopt a different approach for the online farm. Dedicated processes would read files which contain events to be reprocessed. The events would then be sent to the reprocessing tasks on the farm and the reconstructed events would be collected from these ones and stored again in the online storage.

This paper discusses the architecture and implementation of the network file system, or streaming service, which meets the $\mathrm{LHCb}$ experiment requirements.

\section{REQUIREMENTS}

The system has to fulfill the following requirements:

- Events accepted by the HLT farm are sent to a Streaming Layer, made of several Streaming Servers. An event will be sent to a "Stream" according to its characteristics. The same streaming architecture is used for event reprocessing. The throughput depends both on the efficiency of the HLT and on the size of the events written to or read from the storage. Indeed if the rate of accepted event is high, or if the event size is big, the throughput will be higher. The file system must be able to sustain writing and reading of event data simultaneously from several Streaming Servers.

- The file system must be fault tolerant. This means that it should be able to withstand component level failures without any data loss or interruptions of the operation. Recovering from a failure should not result in I/O operations from the Streaming Servers being blocked. In the case of data taking, a write operation blocking would result first in event buffering in the Streaming Layer, which with back-pressure would cause event buffering in the HLT farm. At this point, subsequent incoming events to the HLT farm may be dropped, because its input uses an unreliable protocol [6].

- The system must be scalable to support higher throughput as the needs of the experiment will grow.

${ }^{1}$ To inject a new fill.

${ }^{2}$ For technical maintenance. 


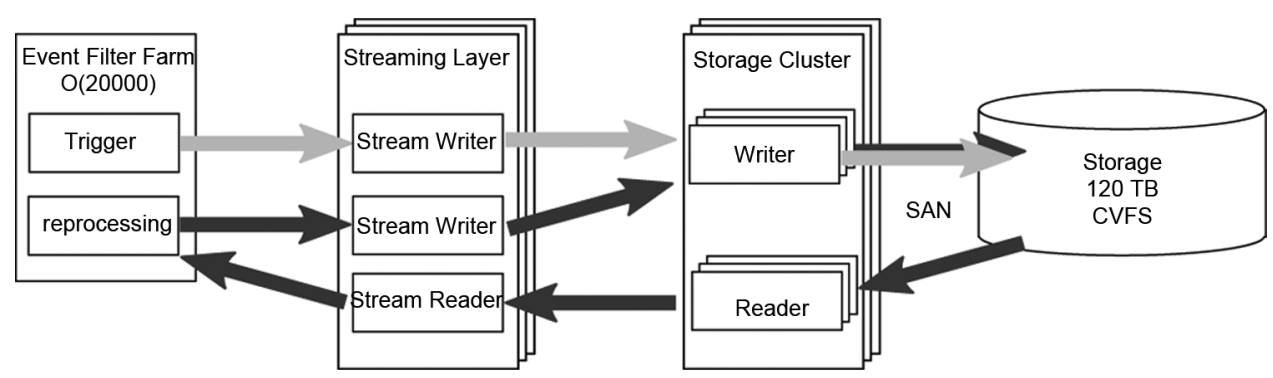

Fig. 1. The LHCb Event Streams. The grey arrows describe the data acquisition use case. The Trigger running on the Event Filter Farm sends accepted events to the Streaming Layer. There the events are sorted according to their characteristics and directed to different Streams. At least one Stream Writer sends Stream data to a Writer Service running on the Storage Cluster. The Writer Service writes to the SAN FS. The black arrows describe the reprocessing. On the storage cluster, Reader Services read files from the SAN FS. On request, they provide chunks to the Stream Readers. Stream Readers distributes the events to the reprocessing tasks running on the Event Filter Farm. Once an event has been reprocessed, it undergoes the same process as accepted events of the data acquisition use case.

\section{ARCHITECTURE}

The LHCb Streaming Service is deployed on a cluster of servers, attached to shared disk storage over a Storage Area Network (SAN). Using a shared disk file system, or SAN File System, enables the entire cluster to access a unified namespace, which offers great flexibility in deployment and scaling of applications. The chosen SAN file system is StorNext File System, previously named CentraVision File System (CVFS) [7]. It is an asymmetric file system, using centralized metadata servers. Like all shared-disk file systems, it uses journals and a lock manager to enable simultaneous access while maintaining file system integrity.

The LHCb Streaming Service is used by two tasks, the data acquisition and the data reprocessing, as shown in Fig. 1. These tasks should not operate simultaneously as the farm usage of each task should be exclusive. It is however to be foreseen as a small slice of the farm could be used for a light reprocessing during a heavy data taking activity. This is ensured by a partitioning mechanism.

A SAN file system simplifies the implementation of a failover mechanism. It is basically possible to have multiple active instances of an application accessing the same file system.

The idea is to export our SAN file system from our Storage Cluster to our Streaming Servers. There are a few technical requirements here, related to the one presented in the previous section. First, every single event reaching the Streaming Servers should be written to disk. It would be preferable to do not suffer event losses at this level. A Streaming Server has to make sure that the event is written out to disk before to free its allocated memory. Second, the throughput has to be maximised in order to make the best use of the SAN file system throughput. It would sustain ideally up to $170 \mathrm{MB} / \mathrm{s}$ per stream, with an average of five streams used. At last, addressing the fault tolerance requirement calls for the use of high availability, and at best the stream load would be balanced amongst the Streaming Servers. A possible solution could have been to run NFS [8] on all the storage cluster, but there are some weaknesses. NFS can be configured to meet our requirement of no data loss. Write requests can be acknowledged once data was written out to disk, but performance penalties are severe. Another configuration allows to have good performance, acknowledging write requests asynchronously. This is however unreliable. Using NFS, an additional issue appears in the underlying file system. If the data are cached in its memory, they can be lost in case of system failure. So the exported file system should make sure that the data are effectively written to disk before to free them from memory. Though a few solutions such as Linux HA (High Availability) [9] and Linux Virtual Server (LVS) [10] allow implementing a clustered NFS solution, there is no commonly availably dynamically load balanced NFS service with failover. NFS does not satisfy our requirements. Hence we have implemented our own network file system to export the SAN file system from the storage cluster servers to the Streaming Servers. It ensures that the cumulative disk throughput is close to the maximum offered by the SAN file system.

Writing successfully every single event asks for a clever failover mechanism. The client of the file system is running on the Streaming Servers. It knows a list of storage cluster nodes on which the file system runs and is ready to respond to commands. It selects one of these nodes and communicate exclusively with it. The communication consists in sending commands to the server, which in return notifies the client that the data were really flushed to the disk. In case of failure of the selected storage server node, the client selects a new node and replays all unacknowledged commands.

The effective write operation is performed once enough events have been received. They are first aggregated into a chunk. Its size corresponds to the optimised chunk size to write on our SAN file system. A write command will therefore not be acknowledged only once the chunk containing its data has been effectively flushed to disk.

Read operations should not miss a single event. Though it is less critical as data is stored, it would then be needed to reprocess the files one more time. Consequently the failover and failback architecture is used as well, so that reading would be as reliable as writing.

It has been chosen to separate completely the tasks of reading and writing in two different multi-threaded services as shown in Fig. 2. This to ensure that both tasks will be completely independent.

\section{WRITER SERVICE IMPLEMENTATION}

For an exhaustive description of the Writer Service, please refer to [9]. Here we will remind the important points and discuss what has changed and what was improved during the production usage of this software. 


\begin{tabular}{|c|c|c|c|c|c|}
\hline $\begin{array}{c}\text { Writer } \\
\text { Discovery }\end{array}$ & $\begin{array}{l}\text { Writer } \\
\text { Failover }\end{array}$ & $\begin{array}{c}\text { Writer } \\
\text { I/O }\end{array}$ & $\begin{array}{c}\text { Reader } \\
\text { Discovery }\end{array}$ & $\begin{array}{l}\text { Reader } \\
\text { Failover }\end{array}$ & $\begin{array}{c}\text { Reader } \\
\text { I/O }\end{array}$ \\
\hline \multicolumn{3}{|c|}{ Writer Service } & \multicolumn{3}{|c|}{ Reader Service } \\
\hline \multicolumn{6}{|c|}{ CVFS File System } \\
\hline
\end{tabular}

SCSI LUNS

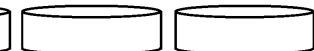

Fig. 2. Software layers of the system. The LUNs are interpreted by CVFS. The Writer and Reader Services running on the Storage Cluster servers export an access to this file system via the I/O thread. Discovery and Failover threads are used for load distribution and high availability.

For data acquisition, the Streaming Server processes are concerned with categorizing the event data into streams. This categorization ends with a process writing over our file system. We henceforth refer to this process as Stream Writer. The server process implementing the file system should be referred as Writer Service.

Events written to disk in the $\mathrm{LHCb}$ experiment have an average size of $35 \mathrm{kB}$, with extremes up to $100 \mathrm{kB}$. In order to achieve good throughput on file systems and disk controllers, write operations should be aligned to precise boundaries such as the page boundary, the sector boundary or the stripe length boundary. Although modern file systems have an efficient page cache architecture to defer write operations to the disk, it has been demonstrated that the page cache algorithms fail to adapt to the streaming nature of the workload in data acquisition systems. One can notice that in programs using page cache in highthroughput sequential I/O, the CPU and memory load generated by memory copy and page cache operation is generally very high. This is mainly due to the copy from userspace to kernelspace. Our file system supports the use of the $O \_D I R E C T$ flag [10] with the open system call. This option simply enables the application to bypass the page cache, to perform Direct Memory Access (DMA) from userspace to the disk. To justify the choice of using $O \_D I R E C T$, tests were performed and are presented in the manuscript [9].

In the Writer Service, all events are aggregated to be aligned to optimal boundaries. The individual operations to the storage controller are performed with an $8 \mathrm{MB}$ chunk size, which provides a good compromise between chunk size and throughput. Using this configurable parameter, we managed to achieve a write throughput of $800 \mathrm{MB} / \mathrm{s}$ over a $10 \mathrm{GbE}$ network. The number of Stream Writers on a single client node is scalable and we can almost reach the line rate, as shown in Fig. 3. We are typically running from two client nodes.

Each Stream Writer opens one or more files at a time and streams all events to these files until they reach a configurable size. Each of these process keeps track of the offset of each event in each file. An event is therefore tagged with a file name and an offset, in order to implement the failover which permits a good reliability in case of file system or hardware failure.

The protocol used to handle the stream is TCP/IP. Then we encapsulate data in an application protocol. We henceforth refer to this protocol as Writer Protocol . It consists of a header which contains a command identifier and the path of the file. The com-

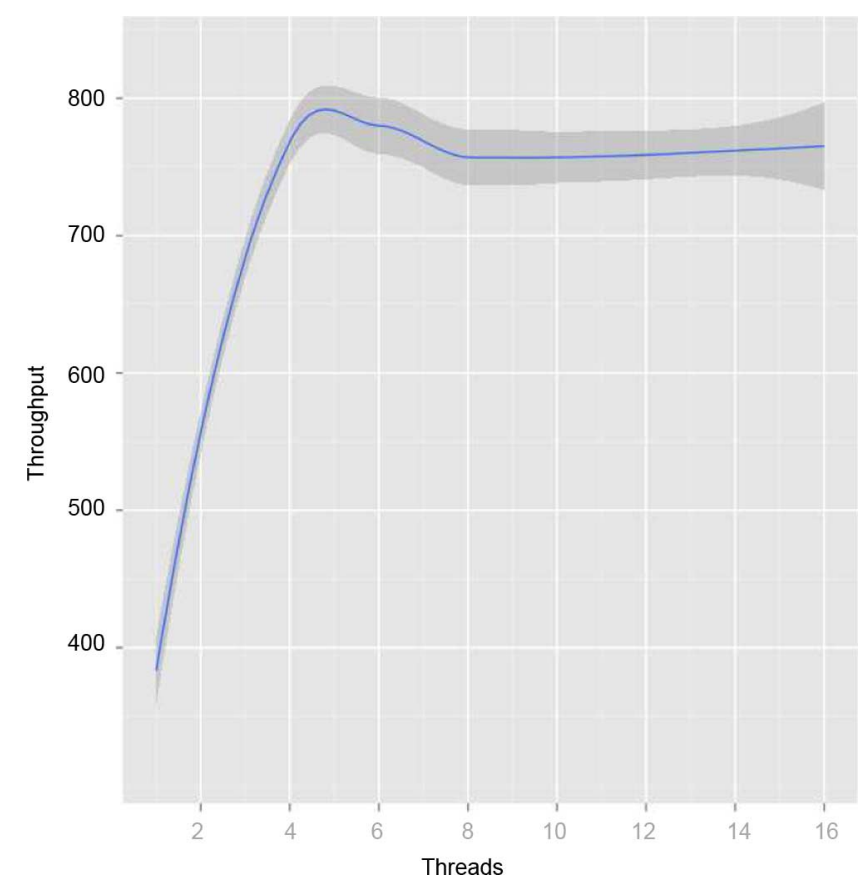

Fig. 3. Write Performance, Throughput in MB/s.

mand identifier tells how to decode the next bytes. It either encodes an open, a close, or a write command, which are encapsulated in a command header. In case of a write command, event data are appended to the command header as a Protocol Data Unit (PDU). The protocol consists of commands and their acknowledgements.

Running the streaming service in production showed an issue in the close command peered with the failover mechanism. As we told, a fail over consists in replaying the commands on another instance of the Writer Service. A close command contains meta data about the files. It is stored in the command. When the command is acknowledged, the information is recorded into a database. In the first implementation, this information was part of the command header. This makes a strong dependency between the Writer Service and the Stream Writers. Any change to the meta data description would imply a change to both of them. As the operation of the experiment became more mature, meta data descriptions started to change. Even a minear change lead both software into a long and exhaustive test process, to be sure that the new version would not bring new bugs. These meta data are used only by the physics application and not by the file system. Their only use there is to be written by Stream Writers into the record data base once the close command is acknowledged. The improvement that has been implemented was simply to move these meta data into a fake PDU of the close command. This PDU is not even sent to the Writer Service, as it would not process it anyway. Once the close command is acknowledged, the Stream Writers can update the data base. Any new meta data information can be added on the Stream Writers without modifying the Writer Service.

\section{READER SERVICE IMPLEMENTATION}

The Reader Service comes as a new process which handles open, read, close, access, stat and seek commands. It already 


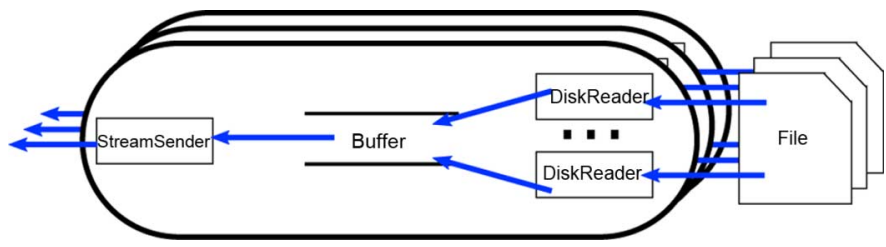

Fig. 4. Multithreaded userspace buffering for file reading.

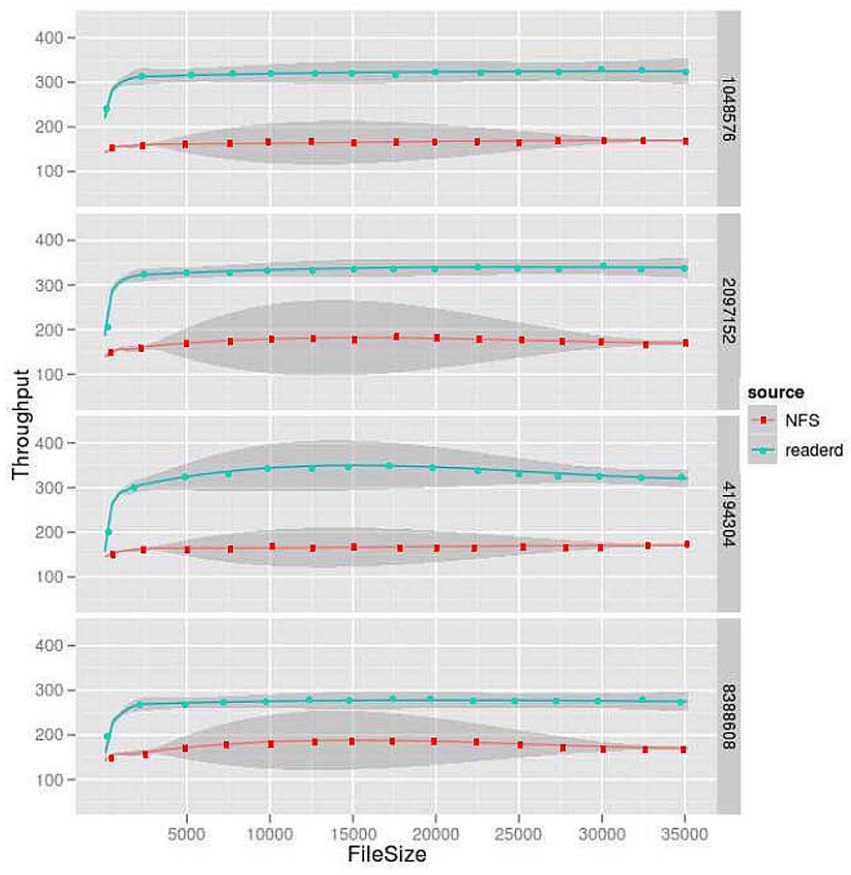

Fig. 5. Performance measurement of our Reader Service, with different configuration, serving a single client. Throughput in MB/s, file size in $\mathrm{kB}$.

adds a lot to our non-POSIX file system, but it is still not POSIX as we are missing a lot of features, like lock. Separating the read and write commands in two different processes is not an issue for us. In our use cases, we do not need to read and write a file from the same process. And if we would need to read a file while another process writes it, the underlying file system would manage this correctly.

The protocol relies once again on TCP/IP. Write commands encapsulate some meta data information which are not mandatory for reading. It is better to strip them out to make the read protocol lighter. Then the protocol does not rely anymore on a command and acknowledgement exchange, but on requests and responses. Responses are in a way acknowledgements which are carrying data, relative to the request. Data from read or stat commands are appended in a PDU of the application protocol.

A good idea of implementing the read command would be to use the sendfile system call which copies data between one file descriptor and another. The copy is performed within the kernel. It is more efficient than a read and write as these would required transferring data to and from the user space. Unfortunately, our underlying file system does not implement sendfile. We have therefore to rely on some userspace buffering.

We therefore studied how to optimize the file reading operation. The two main possibilities are to read it from disk or to map

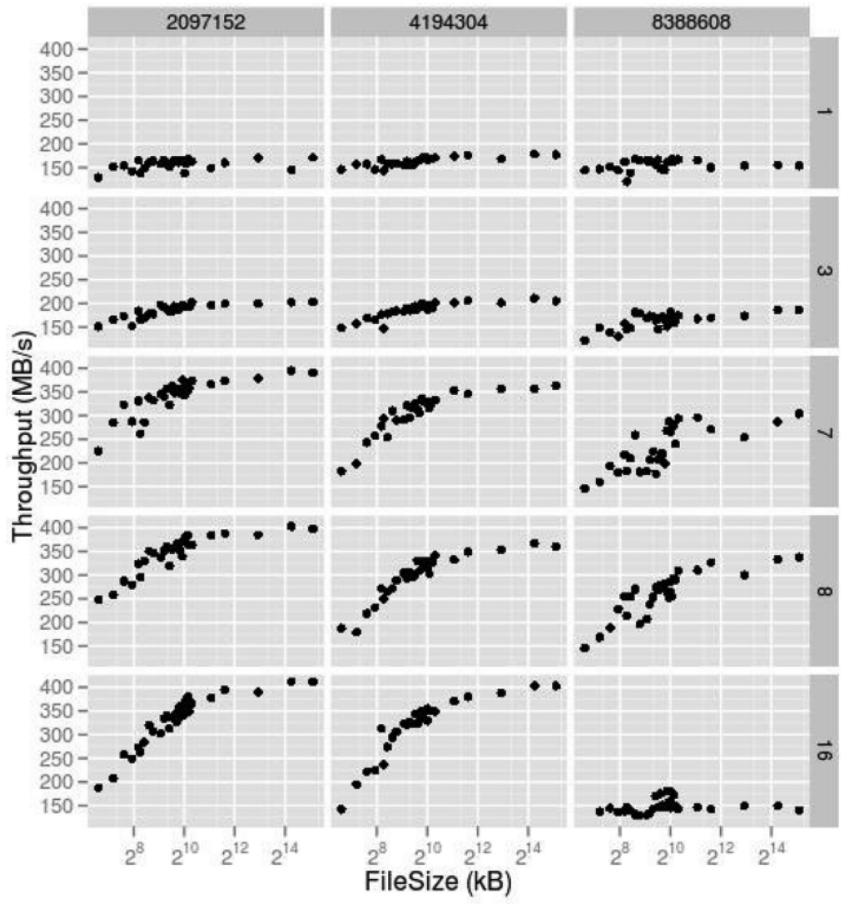

Fig. 6. Performance comparison between the best Reader Service configuration and NFS, serving a single client. Throughput in MB/s, FileSize in kB.

it in memory. The memory mapping solution is not adequate as our files are typically 3 GB large. Due to the large file size, even tuning the operation with posix_madvise was not enough to reach performance of a normal read. Then, focusing on the read call, many comparisons were performed, using $O \_D I R E C T$ or not, tuning the kernel buffering with posix_fadvise.

The chosen solution is to read the file with $O \_D I R E C T$ and to manage the buffering ourselves in the userspace. Several Reader Thread write data from the disk into the buffer and a single Stream Thread receives the requests and sends data from the buffer as responses to the clients. Fig. 4 shows the architecture. Configuration parameters are the chunk size read by each thread and the number of thread, hence the size of the buffer. As the protocol relies on requests and responses, the idea is to cache the data that the user will read before the request comes, implementing read-ahead with a large buffering. The use case is to read files sequentially, from the beginning to the end. The client application here will not read event by event, but a chunk of data in which it will then look for events one by one. Once the file is open, chunks are read from offset zero to a configurable value. On read requests, if the offset required hopefully corresponds to the data already buffered, they can be directly sent to the client. Once the data are sent, a reader thread will read the next chunk in the file and write it into the buffer. If the offset did not correspond to the buffered data, the reader threads will provide the data and anticipate the next ones from this offset.

Tests were performed with a single client reading files from NFS and from our reader service. To give first an idea of the maximum reachable performance, a raw read throughput on the SCSI controller is $1.1 \mathrm{~GB} / \mathrm{s}$. Fig. 5 shows the Reader Service performance for various configuration parameters. The Reader Service was using from one to 16 threads and chunk sizes from 
one to $8 \mathrm{MB}$. The client of the file system was reading chunks of 2,4 and $8 \mathrm{MB}$. The project is still in an early stage and there are some issues. Performance are dropping dramatically when the Reader Service is configured with $8 \mathrm{MB}$ chunks and 16 threads. Fig. 6 shows a comparison between the best configuration of the Reader Service and the best measured configuration of NFS.

Tests have to be performed with several clients in order to measure the scalability of the system and to compare it with the NFS scalability.

\section{CONCLUSION}

We demonstrated that a non-POSIX userspace file system could achieve very good performance and scalability in data writing in comparison to the most common open-source solution and we presented the study and preleminary results of data reading for the same file system design.

\section{ACKNOWLEDGMENT}

The storage system and the Writer Service software architecture was designed by S. S. Cherukuwada.

\section{REFERENCES}

[1] A. J. Augusto Alves et al., The LHCb Detector at the LHC The LHCb Collaboration, Aug. 2008, pp. 1-190 [Online]. Available: http://www. iop.org/EJ/article/1748-0221/3/08/S08005/jinst8_08_s08005.pdf

[2] LHCb HLT The LHCb Collaboration [Online]. Available: http:// LHCb-trig.web.cern.ch/LHCb-trig/HLT

[3] A. Puig Navarro and M. Frank, "Event reconstruction in the LHCb online cluster," in J. Phys.: Conf. Ser., ser. 219, no. 022020.

[4] L. Evans and P. Bryant, LHC Machine Aug. 2008 [Online]. Available: http://www.iop.org/EJ/article/1748-0221/3/08/S08001/ jinst8_08_s08001.pdf

[5] LHC computing Grid, CERN The LCG Editorial Board, 2005, Tech. Rep. CERN-LHCC-2005-024.

[6] B. Jost and N. Neufeld, Raw-Data Transport Format 2004, Tech. Rep. EDMS 499933.

[7] Quantum [Online]. Available: http://www.quantum.com

[8] S. Microsystems, NFS: Network File System Version 3 Protocol Specification 1994 [Online]. Available: www.connectathon.org/nfsv3.pdf

[9] S. S. Cherukuwada and N. Neufeld, "High-performance storage system for the LHCb experiment," IEEE Trans. Nucl. Sci., vol. 55, pp. 278-283, 2008.

[10] A. Arcangeli, O_DIRECT. Manchester: UKUUG, 2001.

[11] The Linux-HA Project. [Online]. Available: http://www.linux-ha.org

[12] The Linux Virtual Server Project. [Online]. Available: http://www.linuxvirtualserver.org 